\title{
Strange probes of Al+Al collisions around threshold
}

\section{Piotr Gasik*, FOPI Collaboration}

University of Warsaw, Faculty of Physics, Hoza 69, 00-681 Warsaw, Poland

E-mail: gasikenpdl. fuw.edu.pl

\begin{abstract}
Strange particles are sensitive probes of hot and dense nuclear matter formed in relativistic nucleus-nucleus collisions. In our experimental studies performed with the FOPI Collaboration at the SIS-18 synchrotron in GSI/Darmstadt, we have measured the $K^{+}$and $K^{-}$yields in $\mathrm{Al}+\mathrm{Al}$ collisions at $1.9 \mathrm{~A} \mathrm{GeV}$. The $K^{-} / K^{+}$ratio is found to be $0.030 \pm 0.007$ (stat.) ${ }_{-0.002}^{+0.004}$ (syst.) and is in good agreement with the previous results of the FOPI, KaoS and HADES Collaborations. The $K^{-} / K^{+}$ratio was also studied as a function of kinetic energy (in CM) of kaons and their rapidity. Different freeze-out conditions can be deduced from the spectra of these particles. This behavior might be related to effect of the KN potentials, which modifies kaon properties inside the medium.
\end{abstract}

50th International Winter Meeting on Nuclear Physics - Bormio2012,

23-27 January 2012

Bormio, Italy

\footnotetext{
* Speaker.
} 


\section{Motivation}

Strange particles are very sensitive probes of hot and dense nuclear matter formed in the relativistic nucleus - nucleus collisions. During such a collision at energies in the range $1-2 A \mathrm{GeV}$ (SIS energy range) the density of nuclear matter in the central region of the collision (fireball) increases up to about 2-3 normal densities. Temperature of the fireball reaches $100 \mathrm{MeV}$. Such conditions last for a very short period of time $(10-20 \mathrm{fm} / \mathrm{c})$ in which new particles are produced. The production probabilities and phase-space distributions of different particle species registered in heavy-ion experiments depend on the conditions reached in the fireball. Thus, they can reflect thermodynamical properties of the hot and dense nuclear matter, as well as theoretically anticipated in-medium modifications of hadrons properties and interactions between them.

\section{1 $K^{+}$and $K^{-}$production at SIS energies}

The production process of kaons is sensitive to nuclear in-medium effects at beam energies below $2 A \mathrm{GeV}$. Due to high threshold-energy for a production in a single $N N$ collision $\left(E_{t h} \approx 1.6\right.$ $\mathrm{GeV}$ for $K^{+}, E_{t h} \approx 2.5 \mathrm{GeV}$ for $K^{-}$), a large number of kaons is produced in multi-step processes during nucleus-nucleus collisions. For example, $\Delta N$ or $\pi N$ reactions contribute substantially to the overall $K^{+}$meson yield [1]. On the other hand, the strangeness exchange reaction $K^{-} N \rightleftharpoons \pi Y$ (where $Y$ stands for $\Lambda$ or $\Sigma$ hyperon) is important in the case of the $K^{-}$production [2]. As a consequence of strangeness-exchange reaction, transport model calculations predict different emission times for $\mathrm{K}^{+}$and $\mathrm{K}^{-}$mesons [3]. This manifests systematically lower inverse slope parameters of the $K^{-}$energy distributions compared to $K^{+}$, as pointed in [3].

One can also interpret the different freeze-out conditions for $K^{+}$and $K^{-}$as a result of another phenomena, e.g.: (i) existence of the $K N$ potentials in nuclear matter and/or (ii) $\phi(1020)$ meson decays which, as reported in [4, 5], significantly influence the total $K^{-}$yield.

\subsection{In-medium modifications of kaons}

It is predicted that in nuclear matter the kaon-nucleon $(K N)$ interaction is modified with respect to vacuum [2]. $K^{+}$mesons are expected to feel a repulsive potential, whereas $K^{-}$mesons should be attracted. As a result, the effective mass and the production threshold energy for kaons should increase slightly, while the corresponding values for anti-kaons should decrease substantially in dense nuclear matter compared to vacuum interactions [6]. The in-medium modifications of kaon properties have been already reported by KaoS [1] and FOPI [7] (experiments focused on strangeness production at SIS-18 energies). Conclusions were based on the comparison to the results of theoretical transport models, however those models seldom consider a potentially important kaon production channel, which involves the production and subsequent decays of $\phi$ mesons.

\subsection{Influence of $\phi(1020)$ meson decays to the $K^{-}$yield}

It should be noted, that the $\phi$ meson mass is only $\sim 30 \mathrm{MeV} / \mathrm{c}^{2}$ larger than the mass of a $\mathrm{K}^{+} \mathrm{K}^{-}$pair. Investigation of $\phi$ meson production may therefore be essential for the conclusions about in-medium effects on kaons, as $\phi$ decays may substantially affect the measured $\mathrm{K}^{-}$yield and phase-space population. 
$\phi$ meson production continues to be the subject of intensive studies at SIS-18 energies. The FOPI Collaboration has measured $\phi$ yield in $\mathrm{Ni}+\mathrm{Ni}$ collisions at $1.93 \mathrm{~A} \mathrm{GeV}$ in two separate experiments $[4,8]$. The number of $\phi$ mesons found in the experimental data samples were respectively 23 and 100 , resulting in the following total $\phi$ yields extrapolated to $4 \pi$ geometry: $(12 \pm 4 \pm 6) \times 10^{-4}$ [4] and $(6 \pm 1 \pm 2) \times 10^{-4}$ [8] per collision. The HADES Collaboration measured $\phi$ meson production on $\mathrm{KCl}$ target irradiated with a $1.756 \mathrm{~A} \mathrm{GeV}$ Ar beam. They found $168 \pm 18 \phi$ mesons in their data sample and obtained a yield $\mathrm{P}(\phi)=\left(2.6 \pm 0.7 \pm 0.1_{-0.3}^{+0.0}\right) \times 10^{-4}$ per collision [5].

The $\phi / K^{-}$ratio has also been estimated. It has been reported that even as much as $20 \%$ of the $K^{-}$mesons produced in $\mathrm{Ni}+\mathrm{Ni}$ collisions at $1.9 \mathrm{~A} \mathrm{GeV}$ beam energy can originate from $\phi$ decays [4]. According to the HADES measurements in the $\mathrm{Ar}+\mathrm{KCl}$ system at $1.756 \mathrm{~A} \mathrm{GeV},(18$ $\pm 7) \% \mathrm{~K}^{-}$originate from $\phi$ decays [5]. This implies, that $\phi$ production contributes significantly to the $K^{-}$yield. One can also estimate, that most of the $\phi$ mesons $(\sim 80 \%)$ produced in such collisions decay outside the fireball. Therefore, $K^{-}$coming from $\phi \rightarrow K^{+} K^{-}$decays, would be produced mostly outside the medium. This effect may reduce any in-medium effects of $K^{-}$as well as substantially affect the $K^{-}$kinetic energy distributions. Precise measurement of $\phi$ production and its influence on the total $K^{-}$yield is therefore essential for drawing conclusions about kaon in-medium production.

The FOPI Collaboration has performed a high-statistics experiment to study strangeness production in $\mathrm{Al}+\mathrm{Al}$ collisions at $1.9 \mathrm{~A} \mathrm{GeV}$ beam kinetic energy. We now report on the measurement of charged kaon production in performed experiment.

\section{FOPI Spectrometer}

FOPI is a modular detector dedicated for the fixed-target experiments at the SIS-18 synchrotron in GSI-Darmstadt. It consists of 4 sub-detectors: CDC, Barrel, Helitron and Forward Plastic Wall (see figure 1). The drift chambers and the plastic Barrel are located within the magnetic field of $0.6 \mathrm{~T}$. This set-up is capable of measuring directly the charged products of the reaction in almost complete $4 \pi$ geometry. The mass of the particle is calculated from magnetic rigidity and energy loss measurements in drift chambers. Plastic detectors give additional information about the time of flight. Matching hits in the ToF detectors with tracks reconstructed in drift chambers enhances mass resolution capabilities. Neutral particles can be identified in the FOPI spectrometer only by reconstructing their invariant masses using charged decay products.

\section{Results}

In the experiment reported in this work, the aluminum target of $702 \mathrm{mg} / \mathrm{cm}^{2}$ thickness was irradiated by the beam of aluminum ions of roughly $8 \times 10^{5} \mathrm{~s}^{-1}$ intensity. The number of collected events was $3 \times 10^{8}$. Centrality of events was determined by the multiplicity of charged particles in the Barrel PlasticWall detectors. The results presented in the following report are based on the most central events, corresponding to $9 \%$ of the total geometrical cross section $(\Delta \sigma \approx 150$ $\mathrm{mb})$. Using the geometrical sharp-cut-off model, one can estimate the maximal value of impact parameter for analyzed data sample to be equal $b_{\text {geo }}^{\max } \approx 1.4 \mathrm{fm}$. The mean number of participants 


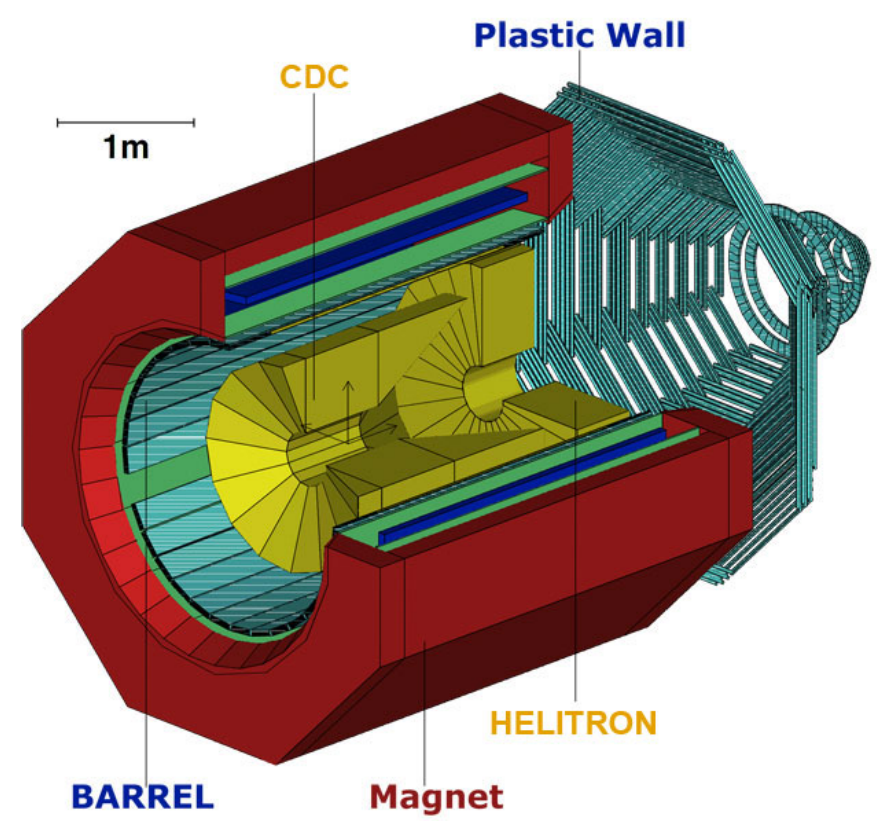

Figure 1: FOPI experimental setup. Barrel and PlaWa (Plastic Wall) are position-sensitive plastic scintillator detectors used for time-of-flight (ToF) and energy loss $(\Delta \mathrm{E})$ measurements. CDC (Central Drift Chamber) and Helitron are multi-wire drift chambers enabling tracking and $\Delta \mathrm{E} / \Delta \mathrm{x}$ measurements.

characterizing the sample of events of which impact parameters are less than $b_{g e o}^{\max }$ was calculated using the participant-spectator model [9] and is equal to $\left\langle A_{\text {part }}\right\rangle \approx 36$.

\subsection{Charged kaons production in $\mathrm{Al}+\mathrm{Al}$ collisions at $1.9 \mathrm{~A} \mathrm{GeV}$}

For the present analysis, the $K^{+}$and $K^{-}$mesons were measured in the CDC and the surrounding plastic Barrel $\left(27^{\circ}<\Theta_{l a b}<57^{\circ}\right)$. Figures $2 \mathrm{a}$ and $3 \mathrm{a}$ show mass distributions obtained for particles with charge +1 and -1 respectively. Cuts applied in the analysis disturbed gaussian shapes of the $\pi^{+}, \pi^{-}$and proton signals, however $K^{+}$and $K^{-}$signals remained undisturbed. $K^{+}$signal, around the mass value of $0.5 \mathrm{GeV} / \mathrm{c}^{2}$, is visible on the tails of the proton and $\pi^{+}$signals, which are described with the combination of gaussian and exponential functions (green line). Background under the anti-kaon peak is caused only by the strong negative pion signal, which is described with the exponential function only. After background subtraction clear peaks around nominal $K^{ \pm}$mass are visible in figures $2 \mathrm{~b}$ and $3 \mathrm{~b}$. Black curves represent gaussian fits. About $190 \times 10^{3}$ of $K^{+}$and $3 \times 10^{3} \mathrm{~K}^{-}$mesons were found in the analyzed data sample.

Analysis of the transverse mass spectra and rapidity-density distributions of measured $K^{ \pm}$ mesons resulted in total $K^{+}$and $K^{-}$yields. In order to estimate the $K^{ \pm}$detection efficiency, simulations that include a realistic description of the detector geometry and resolutions were performed using the GEANT package. The estimate of the total $K^{+}$meson production probability in central $\mathrm{Al}+\mathrm{Al}$ collisions $(\Delta \sigma \approx 150 \mathrm{mb})$ at $1.9 \mathrm{~A} \mathrm{GeV}$ beam kinetic energy is

$$
P_{K^{+}}=\left(3.73 \pm 0.04(\text { stat })_{-0.53}^{+0.25}(\text { syst })\right) \times 10^{-2}
$$



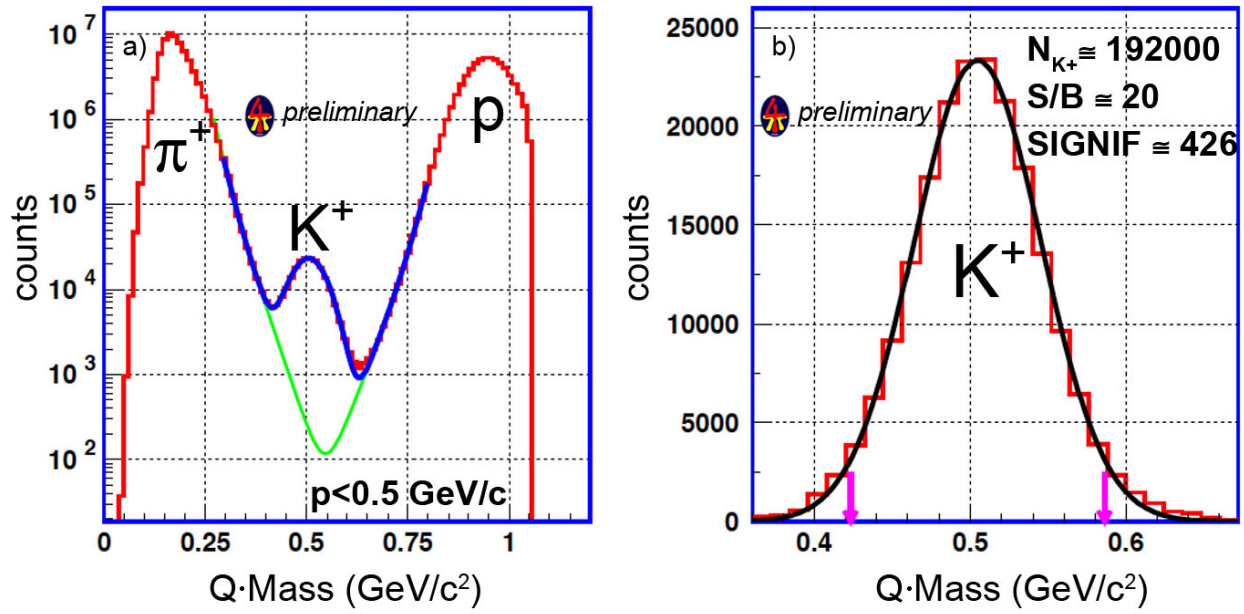

Figure 2: a) Mass spectrum of positively charged particles $(\mathrm{Q}=+1)$ with momentum $p<0.5 \mathrm{GeV} / \mathrm{c} \mathrm{de}$ tected in the spectrometer. The $K^{+}$signal, around the mass value of $0.5 \mathrm{GeV} / \mathrm{c}^{2}$, is visible on the tails of the strong pion and proton signals, which are described as a sum of gaussian and exponential functions (green line). Cuts applied in the analysis disturbed gaussian shapes of the pion and proton signals. The $K^{+}$signal remained undisturbed.

b) The kaon signal after background subtraction. The black curve represents the gaussian fit. About $190 \times 10^{3} K^{+}$mesons were found in this sample. The signal to background ratio (S/B) and significance of the signal (SIGNIF) are also presented.
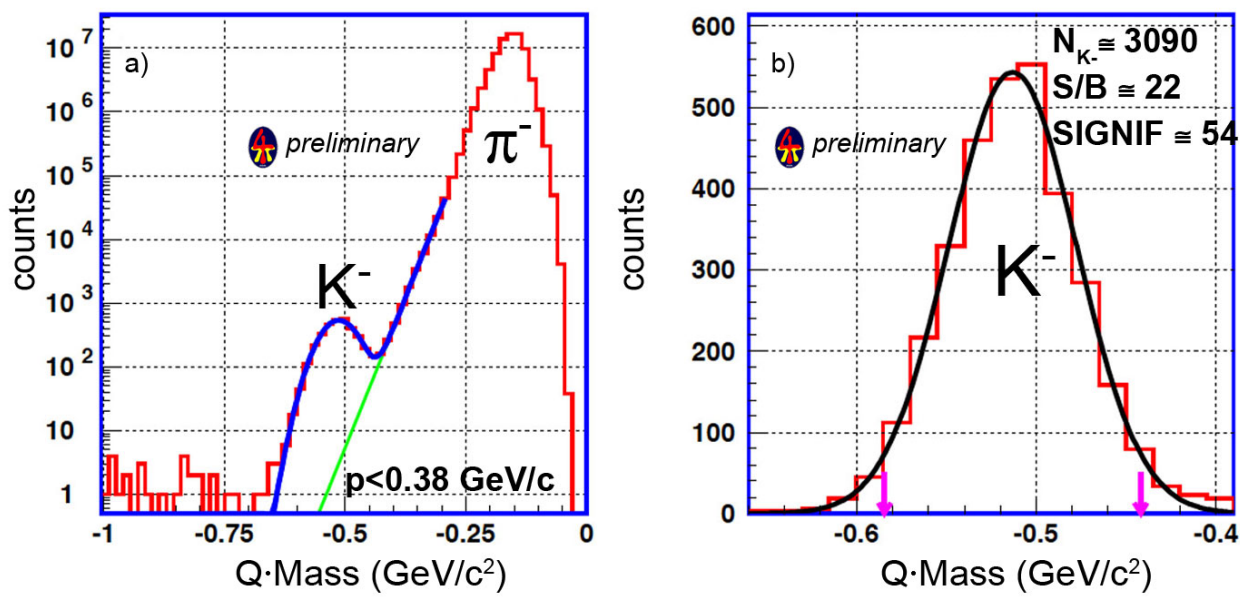

Figure 3: a) Mass spectrum of negatively charged particles $(\mathrm{Q}=-1)$ with momentum $p<0.38 \mathrm{GeV} / \mathrm{c}$ detected in the spectrometer. The $K^{-}$signal, around the mass value of $0.5 \mathrm{GeV} / \mathrm{c}^{2}$, is visible on the tail of the strong pion signal, which is described as an exponential function (green line). Cuts applied in the analysis disturbed gaussian shape of the pion signal. The $K^{-}$signal remained undisturbed.

b) The anti-kaon signal after background subtraction. The black curve represents the gaussian fit. About $3 \times 10^{3} K^{-}$mesons were found in this sample. The signal to background ratio (S/B) and significance of the signal (SIGNIF) are also presented. 

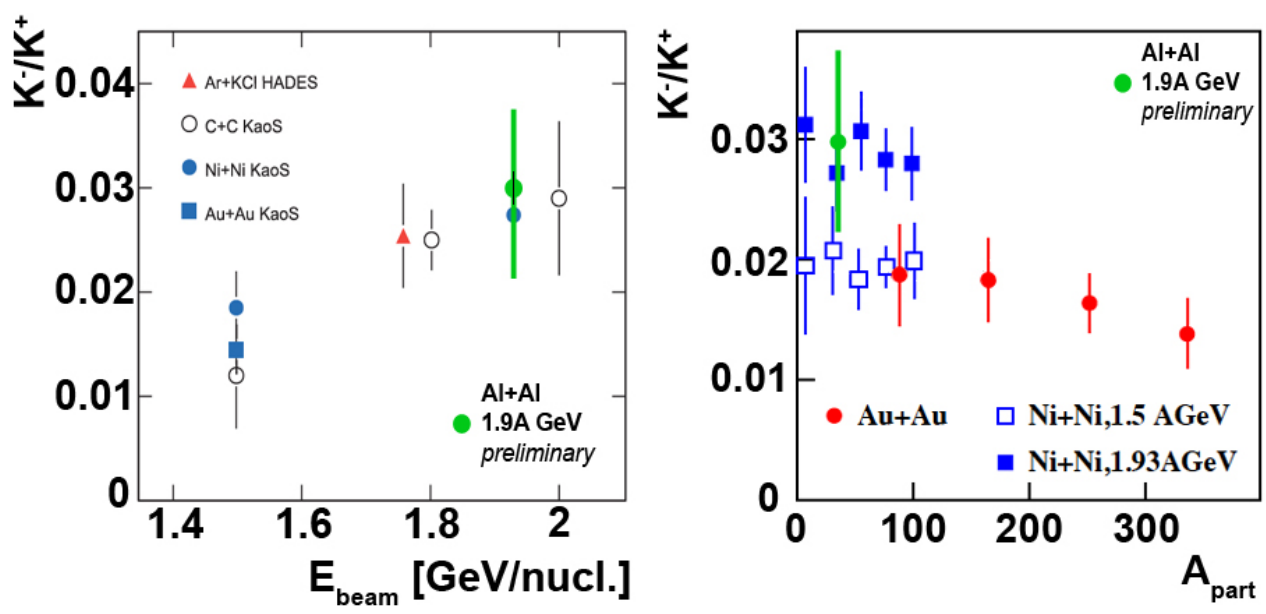

Figure 4: a) $K^{-} / K^{+}$ratios as a function of the kinetic beam energy per nucleon for different systems. Green point depicts FOPI Al+Al result. Picture taken from [5] and modified.

b) $K^{-} / K^{+}$ratios as a function of mean number of participants for different systems and beam energies. Green point depict FOPI Al+Al result. Picture taken from [6] and modified.

whereas the corresponding value for $K^{-}$is equal

$$
P_{K^{-}}=\left(0.11 \pm 0.03(\text { stat })_{-0.02}^{+0.02}(\text { syst })\right) \times 10^{-2} .
$$

Systematic errors indicated above were extrapolated by varying (i) cut conditions, (ii) background subtraction procedure and (iii) centrality conditions in simulated data.

\section{$3.2 K^{-} / K^{+}$ratio}

Dividing the $K^{-}$and $K^{+}$production yields one obtains a $K^{-} / K^{+}$ratio equal to

$$
K^{-} / K^{+}=0.030 \pm 0.007(\text { stat })_{-0.002}^{+0.004}(\text { syst }) \text {. }
$$

This value can be now compared to the results of the other experiments performed on the beam delivered by the SIS- 18 accelerator. Figure 4 shows the $K^{-} / K^{+}$ratios measured as a function of beam energy per nucleon (fig. 4a) and mean number of participants (fig. 4b). Different points show results obtained in different experiments performed by the KaoS and HADES Collaborations (see figure caption for details). Green points indicate the preliminary result reported in this work. $K^{-} / K^{+}$ratio has been also studied as a function of the kinetic energy of kaons and their rapidity. Studying particle yield ratio offers two advantages [7]. (i) Experimental difficulties, like detection efficiencies and acceptance deficiencies, cancel to a large extent. (ii) In-medium effects act in opposite ways on $K^{-}$and $K^{+}$mesons, hence the ratio should reveal these more clearly.

Figure 5a shows the measured $K^{-} / K^{+}$ratio as a function of the kinetic energy in the c.m. reference frame $\left(E_{C M}^{k i n}\right)$ for $\mathrm{Al}+\mathrm{Al}$ collisions. The polar-angle range $136^{\circ}<\Theta_{C M}<150^{\circ}$ has been chosen in order to minimize the edge effects of the $\mathrm{CDC}+$ Barrel subsystem and provide the largest acceptance in $E_{C M}^{k i n}$. The black points indicate experimental data corrected with $K^{ \pm}$detection efficiency. The error bars correspond to the statistical uncertainties. The blue band depicts the variation 
$A l+A l, E=1,9 A ~ G e V$

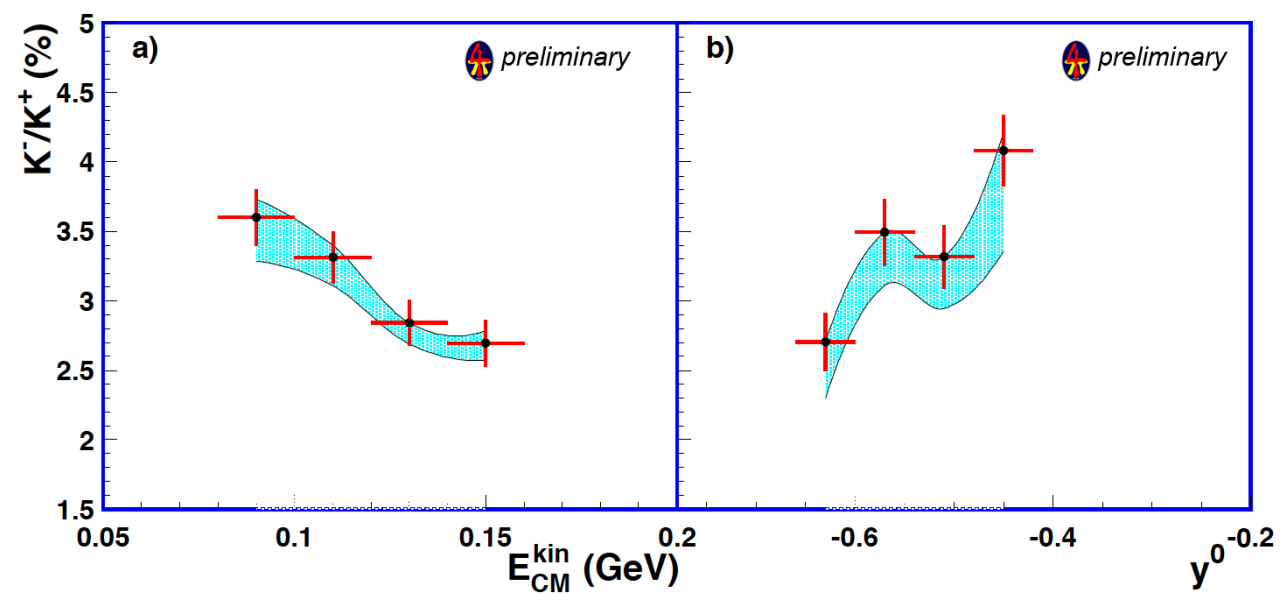

Figure 5: The $K^{-} / K^{+}$ratio as a function of $E_{C M}^{k i n}$ (panel a) and normalized rapidity (panel b) in the $\mathrm{Al}+\mathrm{Al}$ experiment. Error bars represent statistical uncertainties. Light-blue shaded bands represent the estimation of systematical errors.

of the results due to the systematical errors. As shown in figure 5a, the ratio decreases with increasing energy. On average $K^{-}$mesons have lower kinetic energies than $K^{+}$mesons. This suggests that the kinetic energy spectrum of $K^{-}$mesons is softer than the spectrum of $K^{+}$mesons.

In figure $5 \mathrm{~b}$ the $K^{-} / K^{+}$ratio is plotted as a function of the normalized rapidity ${ }^{1}$. The results include particles with momenta $p_{\text {lab }}<0.38 \mathrm{GeV} / \mathrm{c}$. The ratio increases towards mid-rapidity $\left(y^{0}=0\right)$. Again, one can conclude that the $K^{-}$meson final-state momentum-space distribution is more compact than that of $K^{+}$mesons.

Similar results and conclusions have been already reported by FOPI Collaboration in [7], where the analysis of $\mathrm{Ru}+\mathrm{Ru}$ and $\mathrm{Ru}+\mathrm{Zr}$ at $1.69 \mathrm{~A} \mathrm{GeV}$ experiments is shown. Results were compared to the RBUU transport model calculations. It occurred, that only these calculations, which take into account in-medium modifications of the particles, may describe $K^{-} / K^{+}$distributions. The differences between momentum-space distribution of $K^{-}$and $K^{+}$mesons may be therefore explained in the following way. (i) When the in-medium modifications of kaons are taken into account, the repulsive $K N$ potential repels $K^{+}$mesons from the central, dense zone of the collision. (ii) attractive in-medium potential decelerate $K^{-}$mesons during their propagation. In this way, mean kinetic energy of $K^{-}$is lower than that for $K^{+}$mesons, what is observed in the experiment. On the other hand, the kinetic energy distribution of $K^{-}$mesons may be also softened by the contribution of the $K^{-}$coming from $\phi$ decays into the overall $K^{-}$yield. This effect will also reduce any observed in-medium effects of anti-kaons.

\footnotetext{
${ }^{1}$ normalized rapidity $y^{0}=\frac{y}{y^{C M}}-1$, where $y$ is the rapidity of the particle and $y^{C M}$ is the rapidity of the c.m. reference frame
} 


\section{Summary and outlook}

In summary, we report on the measurement of the $K^{-}$and $K^{+}$mesons production probability in $\mathrm{Al}+\mathrm{Al}$ collisions at $1.9 \mathrm{~A} \mathrm{GeV}$. The $K^{-} / K^{+}$ratio was obtained and compared to the $\mathrm{KaoS}$ and HADES data. The result obtained within this work fits well to the results published by those Collaborations. The $K^{-} / K^{+}$ratio was also studied as a function of kinetic energy in the c.m. reference frame and the rapidity of particles. Th final-state momentum-space distribution seems to be narrower for $K^{-}$than for $K^{+}$. The latter has been already reported by the KaoS [6] and FOPI [7] Collaborations.

In the next steps of the analysis, the $K^{-} / K^{+}$ratio distributions should be compared to the transport model calculations to verify, whether any conclusions about in-medium effects on kaons may be drawn. Moreover, the $\phi(1020)$ meson contribution to the total $K^{-}$yield should be also considered. Results will be reported soon by the FOPI Collaboration.

\section{FOPI Collaboration}

Anton Andronic ${ }^{4}$, Ralf Averbeck ${ }^{4}$, Valerie Barret ${ }^{3}$, Zoran Basrak ${ }^{16}$, Nicole Bastid ${ }^{3}$, Mohammed Lotfi Benabderrahmane ${ }^{6}$, Martin Berger ${ }^{10}$, Paul Bühler ${ }^{14}$, Roman Caplar ${ }^{16}$, Ivana Carević ${ }^{12}$, Michael Cargnelli ${ }^{14}$, Olga Cherviakova ${ }^{15}$, Mircea Ciobanu ${ }^{4}$, Philippe Crochet $^{3}$, Ingo Deppner ${ }^{6}$, Pascal Dupieux ${ }^{3}$, Mile Dželalija ${ }^{12}$, Laura Fabbietti ${ }^{10}$, Arnaud Le Fèvre ${ }^{4}$, Zoltan Fodor ${ }^{2}$, Jochen Frühauf $^{6}$, Piotr Gasik ${ }^{15}$, Igor Gašparić ${ }^{16}$, Yuri Grishkin ${ }^{8}$, Olaf Hartmann ${ }^{14}$, Norbert Hermann ${ }^{6}$, Klaus Dieter Hildenbrand ${ }^{4}$, Byungsik Hong ${ }^{11}$, Tae Im Kang ${ }^{6}$, Jozsef Kecskemeti ${ }^{2}$, Young Jin $\mathrm{Kim}^{4}$, Paul Kienle ${ }^{14}$, Marek Kirejczyk ${ }^{15}$, Mladen Kišs ${ }^{4,16}$, Roland Kotte ${ }^{5}$, Piotr Koczoń ${ }^{4}$, Alexander Lebedev ${ }^{8}$, Yvonne Leifels ${ }^{4}$, Pierre-Alain Loizeau ${ }^{6}$, Xavier Lopez ${ }^{3}$, Vladislav Manko9 ${ }^{9}$ Johann Marton ${ }^{14}$, Tomasz Matulewicz ${ }^{15}$, Markus Merschmeyer ${ }^{6}$, Robert Münzer ${ }^{10}$, Mihai Petrovici ${ }^{1}$, Krzysztof Piasecki ${ }^{15}$, Dominik Pleiner ${ }^{10}$, Fouad Rami ${ }^{13}$, Willibrord Reisdorf ${ }^{4}$, Ming Sang Ryu ${ }^{11}$, Andreas Schüttauf ${ }^{4}$, Zoltan Seres ${ }^{2}$, Brunon Sikora ${ }^{15}$, Kwang Souk Sim ${ }^{11}$, Victor Simion ${ }^{1}$, Krystyna Siwek-Wilczyńska ${ }^{15}$, Vladimir Smolyankin ${ }^{8}$, Ken Suzuki $^{14}$, Zbigniew Tymiński ${ }^{15}$, Eberhard Widmann ${ }^{14}$, Jakob Wierzbowski ${ }^{10}$, Krzysztof Wiśniewski ${ }^{6,15}$, Zhi Gang Xiao ${ }^{7}$, Hu Shang Xu ${ }^{7}$, Igor Yushmanov ${ }^{9}$, Xue Ying Zhang ${ }^{7}$, Ya Peng Zhang ${ }^{6}$, Alexander Zhilin ${ }^{8}$, Johann Zmeskal ${ }^{14}$, Victoria Zinyuk ${ }^{6}$.

${ }^{1}$ NIPNE Bucharest, ${ }^{2}$ KFKI RMKI Budapest, ${ }^{3}$ LPC Clermont-Ferrand, ${ }^{4}$ GSI Darmstadt, ${ }^{5}$ FZ Dresden-Rossendorf, ${ }^{6}$ Universität Heidelberg, ${ }^{7}$ IMP Lanzhou, ${ }^{8}$ ITEP Moscow, ${ }^{9}$ KI Moscow,

${ }^{10}$ Technische Universität München, ${ }^{11}$ Korea University Seoul, ${ }^{12}$ University of Split, ${ }^{13}$ IPHC Strasbourg, ${ }^{14}$ SMI Vienna, ${ }^{15}$ University of Warsaw, ${ }^{16}$ RBI Zagreb,

\section{References}

[1] C. Fuchs, Prog. Part. Nucl. Phys. 56 (2006) 1

[2] C.M. Ko, G.Q. Li, J. Phys. G 22 (1996) 1673

[3] A. Förster et al., Phys. Rev. C75 (2007) 024906

[4] A. Mangiarotti et al., Nucl. Phys. A 714 (2003) 89 
[5] G. Agakishiev et al., Phys. Rev. C 80 (2009) 025209

[6] J. Schaffner-Bielich, J. Bondorf, I. Mishustin, Nucl. Phys. A 625 (1997) 325

[7] K. Wisniewski et al., Eur. Phys. J. A 9 (2000) 515

[8] Z. Tymiński, Ann. Rep. of Nucl. Phys. Div. IEP 2004, p. 13, Warsaw University

[9] J. Gosset et al., Phys. Rev. C16 (1997) 629 